OPEN

SUBJECT AREAS:

FLUID DYNAMICS

CHEMICAL PHYSICS

APPLIED PHYSICS

MICROFLUIDICS

Received

26 March 2013

Accepted

3 June 2013

Published

20 June 2013

Correspondence and requests for materials should be addressed to D.J.I. (kennyi@postech. ac.kr) or K.H.K. (khkang@postech.ac.

$\mathrm{kr})$

* These authors contributed equally to this work.

\section{Spontaneous electrical charging of droplets by conventional pipetting}

\author{
Dongwhi Choi' *, Horim Lee ${ }^{1 *}$, Do Jin Im², In Seok Kang² $^{2}$ Geunbae Lim', Dong Sung Kim \\ \& Kwan Hyoung Kang'
}

\begin{abstract}
'Department of Mechanical Engineering, Pohang University of Science and Technology, San 31 Hyoja-dong, Nam-Gu, Pohang, Gyeongbuk, 790-784, South Korea, ${ }^{2}$ Department of Chemical Engineering, Pohang University of Science and Technology, San 31 Hyoja-dong, Nam-Gu, Pohang, Gyeongbuk, 790-784, South Korea.
\end{abstract}

We report that a droplet dispensed from a micropipette almost always has a considerable electrical charge of a magnitude dependent on the constituents of the droplet, on atmospheric humidity and on the coating material of pipette tip. We show that this natural electrification of a droplet originates from the charge separation between a droplet and pipette tip surface by contact with water due to the ionization of surface chemical groups. Charge on a droplet can make it difficult to detach the droplet from the pipette tip, can decrease its surface tension, can affect the chemical characteristics of solutions due to interactions with charged molecules, and can influence the combination and localization of charged bio-molecules; in all cases, the charge may affect results of experiments in which any of these factors is important. Thus, these findings reveal experimental parameters that should be controlled in experiments that use micropipettes.

M icropipettes that dispense an adjustable volume of liquid from a disposable tip are commonly used in medicine, biology, chemistry, and microfluidics ${ }^{1-3}$. Despite their frequent use in many scientific and engineering fields, the fact that a micropipette almost always dispenses charged droplets is seldom recognized. Pipette tip manufacturers have only reported that the static charge caused by the triboelectric effect between pipette tips and their rack can cause the tips to adhere to a robotic instrument's head in automated systems ${ }^{4}$. Recently, our group observed the presence of charge on a dispensed droplet and did not know from where the charge is originated ${ }^{5,6}$.

If a pipette tip is left close to a droplet after dispensing it in oil, the droplet does not fall down but is pulled toward the tip (Figure 1a; Supplementary Video S1). When droplets are dispensed in oil consecutively, they do not coalesce but repel each other (Figure 1b; Supplementary Video S2). When a droplet is dispensed near a metallic plate immersed in oil without electrical connections, the droplet is attracted to the plate (Supplementary Video S3). These observations can be explained by the electrostatic effect or the image charge effect and are consistent with the hypothesis that droplets dispensed by pipetting are electrically charged.

In this paper, we report the electrification of the droplets dispensed from pipette tips. We examined how much charge a droplet acquires from the pipette tip. The amount of charge on the droplet was precisely measured using the Faraday cup (FC) methods or the droplet trajectory (DT) method, in which analysis of droplet motion was used to infer the charge on it. Parameters that affect the amount of the charge were determined; these include solution conditions such as $\mathrm{pH}$ and concentration of electrolytes, atmospheric humidity, and the coating material of pipette tip. Results suggest that the electrification of droplets by pipetting is caused by the ionization of surface chemical groups on the pipette tip. Finally, we discuss the implications of the present findings in scientific and engineering experiments.

\section{Results}

The existence and measurement of the charge of dispensed droplets from pipette tips. The existence of the charge of a droplet dispensed from a pipette tip can be simply confirmed by applying a uniform electric field to the droplet, which then shows electrophoretic motion. The polarity of the charge can be checked by changing the direction of the electric field. From the electrophoretic motion, the amount of the charge of the dispensed droplet can be measured using image analysis (Figure 2a) because the combination of gravity and the electric field cause the motion of the droplet to deviate from vertical (Figure 2a, inset; Supplementary Video S4). The charge can be estimated from the force balance between electric and hydrodynamic forces. The hydrodynamic drag force 
a

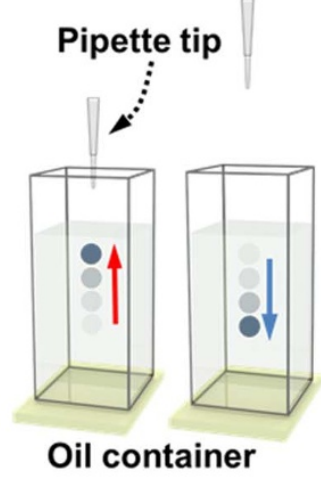

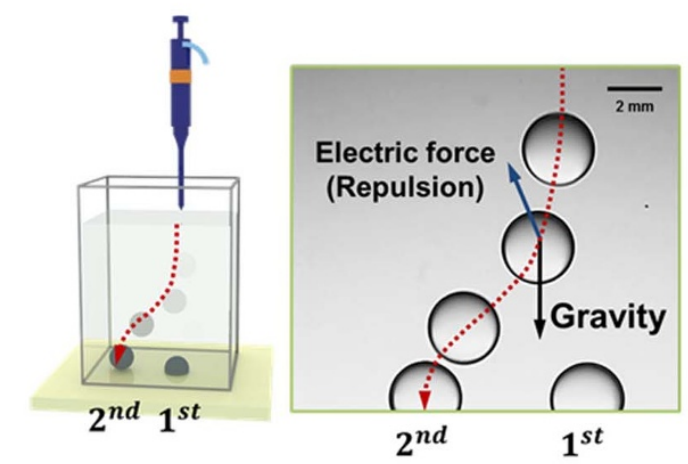

Figure 1 Simple experiments on the existence of a droplet charge. (a) Attractive force occurs between a pipette tip and a dispensed water droplet. (b) When a second droplet is dispensed, the repulsion between the two droplets prevents coalescence.

of a droplet is calculated from its velocity and is decomposed to horizontal $\left(\mathrm{F}_{\mathrm{Dx}}\right)$ and vertical $\left(\mathrm{F}_{\mathrm{Dy}}\right)$ components. By equating the horizontal drag force $\left(\mathrm{F}_{\mathrm{Dx}}\right)$ with the electric force $\left(\mathrm{F}_{\mathrm{E}}\right)$, we can estimate the charge of a droplet.

Results obtained using the DT method agreed well with those obtained using the FC method (Figure 2b,c). In this work, the DT method was primarily used due to the difficulties of transferring a small droplet from a pipette tips to the Faraday cup in air without leaving a residual droplet in the tip; the FC method was used when the DT method was inapplicable.

The deionized water droplets dispensed from pipette tips had a large positive charge of $\sim 10^{-10} \mathrm{C}$ (Figure $2 \mathrm{c}$ ), which is comparable to the charge acquired by direct charging with an electrified electrode under electric field of $3 \mathrm{kV} / \mathrm{cm}^{7}$. The charge amount was positively correlated with the size of droplets (Figure 2c). Charge was also detected in various other aqueous droplets (electrolyte, buffer, acid and base, bio-macromolecule solution (DNA, protein, enzyme), cell suspension, coffee, fruit juice, and milk) (Supplementary Table S1).

The origin of the charge. The charge amount was nearly proportional to the contact area A (calculation details in SI) with the inner surface of the pipette tip (Figure 3); this result suggests that the electrification of the droplets by pipetting is a surface-related phenomenon.

The charge can develop during pipetting by at least two mechanisms. The first is that static charge can accumulate on the pipette tip before aspirating the liquid. Pipette tips can hold a static charge due to various frictions encountered during delivery. If negative static charges develop on a pipette tip surface, more positive ions than negative ions are collected from the solution during aspiration to satisfy electrical neutrality. As a result, the solution inside the pipette tip develops a net positive charge which is transferred to the droplet dispensed from the tip. The second possible source of charge is electrification of the inside surface of pipette tips by contact with water due to ionization of surface groups ${ }^{8,9}$. If a negative surface charge develops on the pipette tip surface due these mechanisms, a positively charged electrical double layer (EDL) would be created to maintain electrical neutrality ${ }^{10}$. Then, when the solution is dispensed as a droplet, the positive charge in the EDL can be carried away with the liquid and the negative charge can remain on the surface of the pipette tip.

To clarify the mechanism by which pipetting electrifies dispensed droplets, the FC method was used to measure charges on a pipette tip and droplet during consecutive steps in the droplet dispensing process (Figure 4). A pipette tip itself had negative charge before fitting it to a pipette (Stage I), but the amount $\left(\sim 10^{-11} \mathrm{C}\right.$ ) was much smaller than that of the droplet $\left(\sim 10^{-10} \mathrm{C}\right)$ dispensed from the pipette tip. The charges of the pipette and pipette tip after fitting (Stage II) and aspirating water (Stage III) were still smaller than that of the dispensed droplet, although the amount of the charge varied slightly; these differences mean that previously developed charge did not affect the electrification of the droplet much. Therefore, we conclude that static charge is not the main mechanism of droplet electrification.

When the droplet was dispensed from the pipette tip, clear charge separation resulted (Stage IV). The amount of charge on the droplet was similar to that on the pipette tip $\left(\sim 10^{-10} \mathrm{C}\right)$ but had opposite sign. This result corresponds to the expectation based on surface electrification due to ionization of surface groups. However, more experiments must be conducted to confirm that this is the electrification mechanism.

To locate the charge when a pipette tip contains water, an electric field was applied inside the pipette tip (Figure 5a). A circulating flow resulted (Figure 5b; Supplementary Video S5) that is similar to electroosmotic flow in a closed microchannel ${ }^{8}$. Electroosmotic flow occurs due to surface charge at the interface between a solid and liquid. The flow pattern was similar to the result of the simplified numerical analysis (inset, Figure 5a) of the electroosmotic flow originating from ad hoc negative surface charge of the pipette tip. These results indicate that the pipette tip surface is negatively charged.

The amount of the surface charge caused by ionization of surface groups is strongly affected by the $\mathrm{pH}$ of the electrolyte solution ${ }^{8,9,11}$. To determine the effects of solution $\mathrm{pH}$ on the charge of a droplet, electrolytes were prepared to minimize effects caused by the type and concentration of the electrolyte solution. The $\mathrm{pHs}$ of potassium chloride $(\mathrm{KCl})$ solutions were regulated using $\mathrm{HCl}$ and $\mathrm{KOH}$ solutions of the same concentration as that of $\mathrm{KCl}$ solutions. This enables preparation of solutions with various $\mathrm{pHs}$ without changing the types of major ions and the concentrations of the electrolyte solutions. The amount of charge showed strong $\mathrm{pH}$ dependence (Figure 5c). Furthermore, the $\mathrm{pH}$ dependence was very similar to that of general surface electrification. Generally, the negative charge developed on a surface increases with the $\mathrm{pH}$ of the solution ${ }^{8,9,11}$. This principle suggests that the negative charge on the inner surface of a pipette tip increases as the $\mathrm{pH}$ of the solution increases, and that as a consequence the positive charge in the water phase increases, as in the present experimental results. Together, these experimental results indicate that the main origin of the electrification of the droplet during pipetting is the interfacial charge due to the ionization of the surface chemical groups on a pipette tip, rather than to frictional static charge which exists before aspiration.

Other parameters affecting the amount of charge. Other parameters also affected the amount of charge, including the concentration of electrolyte solution, the atmospheric humidity, and the coating material of pipette tip inner surface. 
a

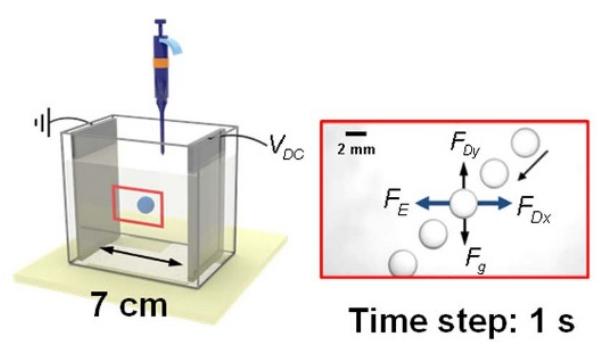

b

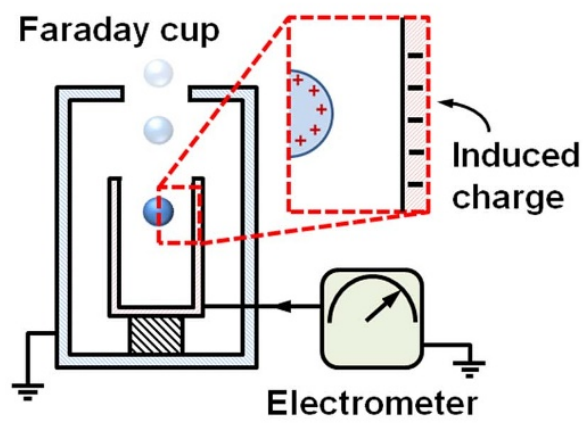

C

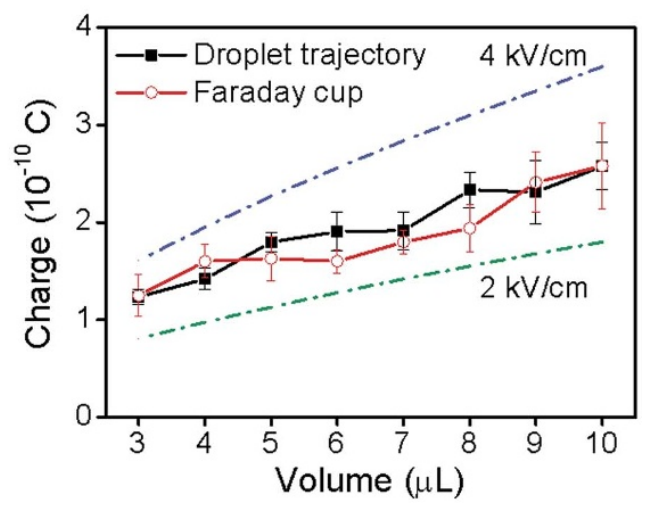

Figure $2 \mid$ Charge on water droplets dispensed from micropipettes.

(a) Schematic of droplet trajectory method for measuring the charge and movement of a charged water droplet dispensed from a micropipette. $F_{D y}$, $\mathrm{F}_{\mathrm{Dx}}, \mathrm{F}_{\mathrm{E}}$, and $\mathrm{F}_{\mathrm{g}}$ denote the vertical, horizontal components of the hydrodynamic drag force, the electric force, and the gravitational force, respectively. The charge is estimated from the droplet velocity.

(b) Schematic of the Faraday cup method for measuring the charge. The Faraday cup is connected to an electrometer and the charge of a droplet is calculated by integrating the current induced in the cup. (c) Mean measured charge $(n=20$, bars \pm s.d.) of a deionized water droplet at applied voltage 0.3 to $0.7 \mathrm{kV}$, relative humidity ( $\mathrm{RH}) 50 \%$ and $\mathrm{pH}=7.4$. Relative standard deviation (RSD) ranges from 7.2 to $14.2 \%$ for the DT method and from 6.7 to $17.2 \%$ for the Faraday cup method. Blue and green lines: maximum theoretical charge of a droplet when it contacts an electrified planar electrode under each electric field.

Electrolyte concentration was negatively correlated with the charge of an electrolyte droplet (Figure 6a). The concentration effect on surface charge of the observed magnitude is unusual in surface chemistry because although the zeta potential is inversely proportional to the square root of concentration, surface charge density is not affected much by concentration ${ }^{11}$.

To investigate the effect of the coating material of pipette tips (Figure $6 \mathrm{~b}$ ), the inner surface of a pipette tip was coated with various

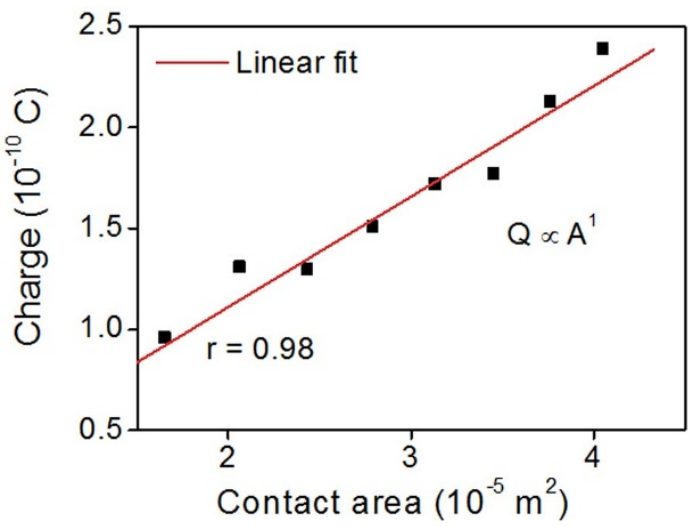

Figure 3 The relationship between the droplet charge $Q$ and the contact area A with pipette tip inner surface. Q is almost proportional to A. (for the linear plot, the correlation coefficient is $\mathrm{r}=0.98$ ).

hydrophobic materials such as polydimethylsiloxane (PDMS), Teflon (AF1600), and silane (Trichloro(3,3,3-trifluoropropyl)silane).

Humidity had a relatively weak effect on droplet charge (Figure 6c). The droplets had slightly less charge when dispensed at $\mathrm{RH}=30 \%$ than at $\mathrm{RH}=50$ and $70 \%$, and the difference between $50 \%$ and $70 \%$ cases was slight. This feature implies again that the static charge is not the main origin of the electrification of droplets

\section{a}
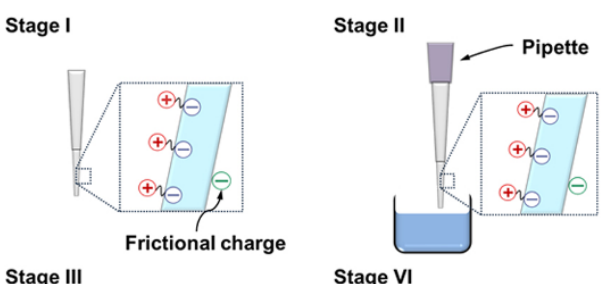

Stage III

Stage VI
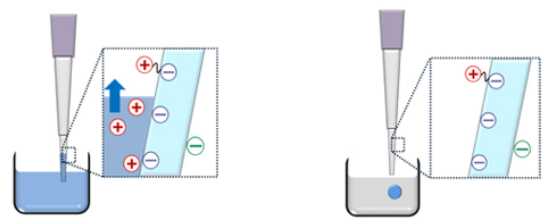

b

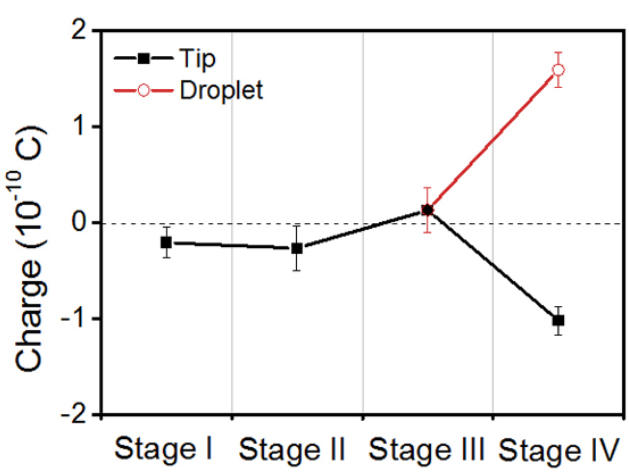

Figure $4 \mid$ Consecutive measurements of the charge during a droplet transferring process. (a) Schematic explanations on the transferring processes. (b) Measured charges of a pipette tip and a 7- $\mu \mathrm{L}$ deionized water droplet $(\mathrm{RH}=50 \%)$. In stage IV, the measured charges of the droplet and the tip are of similar magnitude but opposite sign. 
a

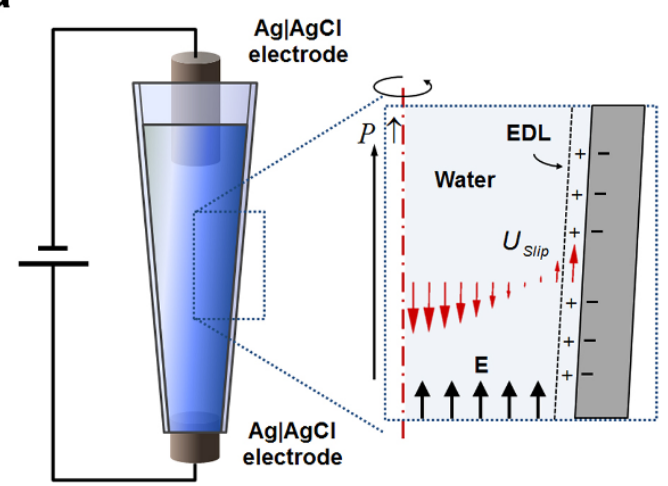

b

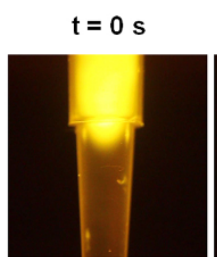

$3 \mathrm{~s}$
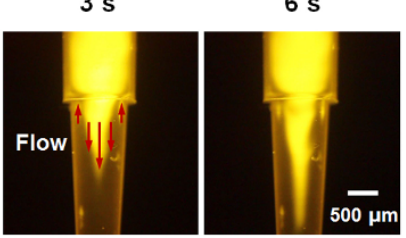

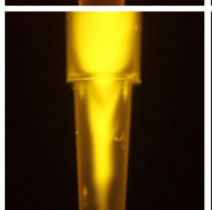

$9 \mathrm{~s}$

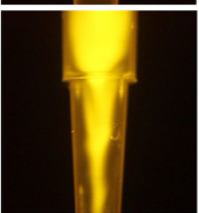

$12 \mathrm{~s}$

C

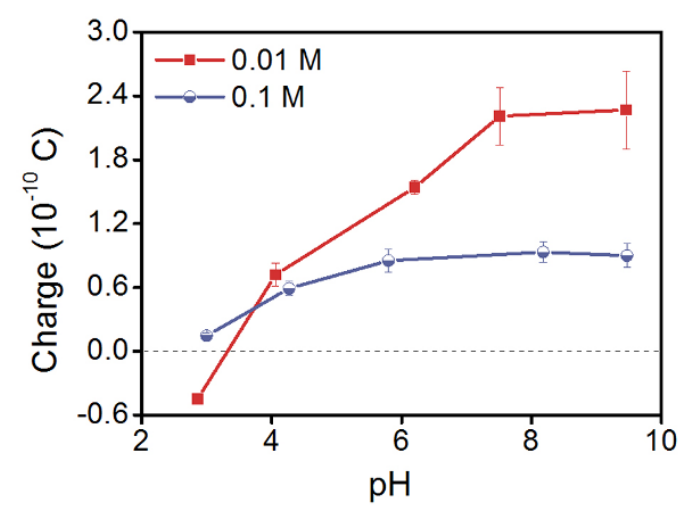

Figure $5 \mid$ Visualization of electroosmotic flow inside a pipette tip and the effect of $\mathrm{pH}$ on the charge of a droplet. (a) Numerical modeling of the electroosmotic flow (b) Electroosmotic flow visualization using fluorescent dye (Rhodamine 6G). $\mathrm{Ag} \mid \mathrm{AgCl}$ electrodes were used to avoid electrolysis; distance between electrodes $18 \mathrm{~mm}$; applied voltage $30 \mathrm{~V}$.

(c) Effects of $\mathrm{pH}$ on charges of $7-\mu \mathrm{L}$ droplets $(\mathrm{RH}=50 \%)$.

because frictional electrification is usually higher at low humidity than at high humidity.

These results for the effects of the parameters, the concentration of electrolyte solution, the atmospheric humidity, and the coating material of pipette tip inner surface, would be helpful information for pipette users. However, further research is needed to explain the reasons for the effects of the parameters.

\section{Discussion}

Results of the experiments suggest that the origin of the electrification of dispensed droplets from a pipette tip is surface charge caused by ionization of the surface chemical groups on a pipette tip. However, details of how the charge is transported have not yet been a
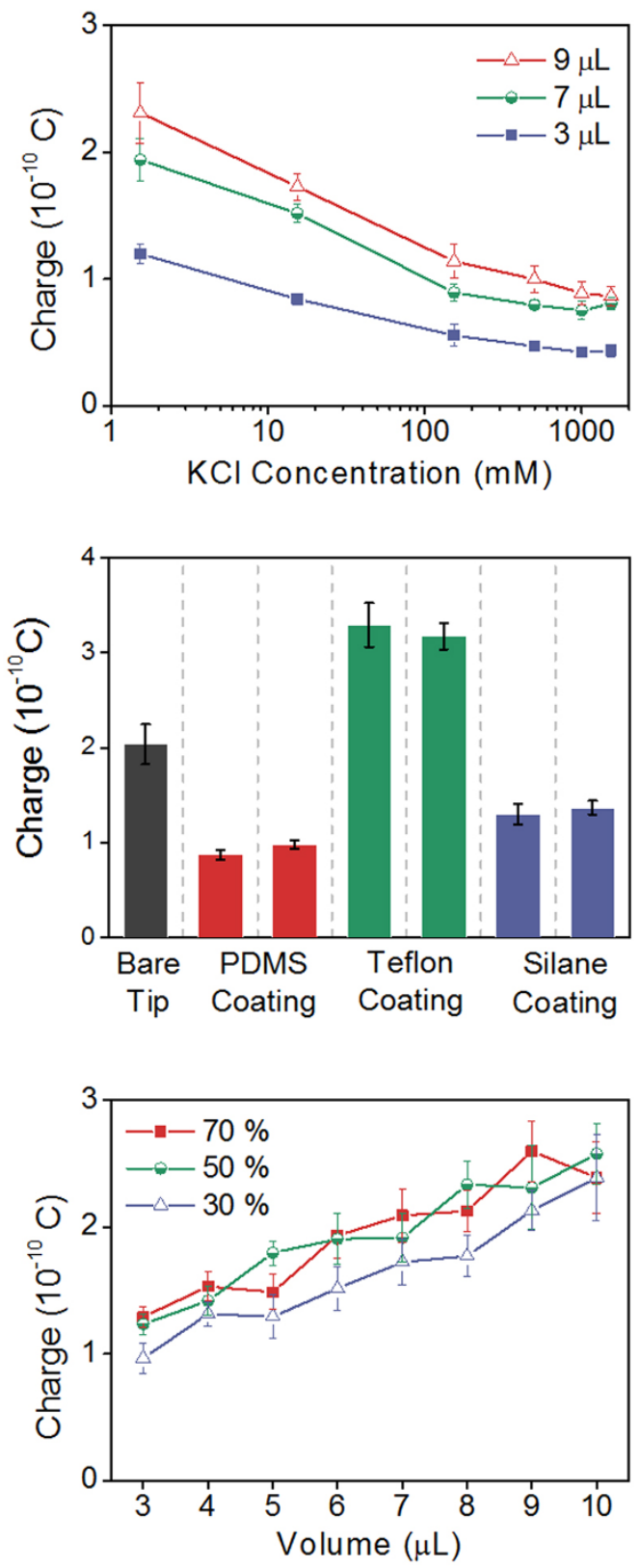

Figure 6 | Effects of electrolyte concentration, coating material of pipette tip inner surface and $\mathrm{RH}$ on the charge of a droplet. (a) Effects of $\mathrm{KCl}$ concentrations $(\mathrm{pH}=7.4, \mathrm{RH}=50 \%$ ). (b) Effects of coating material of pipette tip inner surface (deionized water, dispensed volume $=7 \mu \mathrm{L}, \mathrm{RH}$ $=50 \%)$. The inner surface of a pipette tip was coated with various hydrophobic materials such as polydimethylsiloxane (PDMS), Teflon (AF 1600) and silane (Trichloro(3,3,3-trifluoropropyl)silane). (c) Effects of $\mathrm{RH}$ (deionized water).

discussed, especially the charge transport near the three-phase contact lines. During dispensing, charge separation between the pipette tip and the dispensed droplet occurs and an electrostatic attractive force would occur between the separated charges. This force would cause the charges in the water phase to be concentrated near the three-phase contact lines. The attractive force and charge density at the contact lines may increase during descent of the level of the liquid in the pipette tip, because of the increase of the amounts of the separated charges. This is an interesting feature of the present charge transport. Charge transport during pipetting can be regarded as 
analogous to streaming current, i.e., the charge transport generated by pressure-driven flow in a pipe. However, in a streaming current, the surface charge is always screened by the following charge and is not exposed to air, so that the separated surface charges do not affect each other ${ }^{10}$. This difference leads us to consider the behavior of the charges at the contact lines.

Here, the behavior of the charges in water phase has two possible explanations. First, the charges can be totally transferred with the liquid during dispensing. In electrolyte solutions, charges result from the imbalance of positive and negative ions. Because the ions are confined in the water phase, the charges can be transferred without residual charges during dispensing. Second, a portion of the charges may remain behind on the surface of the pipette tip; this can be due to the existence of liquid film and the strong interaction between the separated charges. A liquid film is formed when a three-phase contact line is receding; thickness of this film is several tens of nanometers, which is comparable with the thickness of the $\mathrm{EDL}^{12,13}$. Therefore, if a charged liquid film is formed during dispensing and is disconnected from the bulk of the liquid, the charge in the liquid film cannot transfer to the liquid droplet. Meanwhile, the strong interaction between the separated charges may cause a deformation of the menisci near the contact lines ${ }^{14,15}$. The deformation may enhance the formation of the liquid film, electromechanically ${ }^{13}$. If the electrical interaction is strong enough to overcome capillary force, it can make the charge escape from the water phase as electrospray ${ }^{16}$.

The magnitude of charge on the droplet was proportional to the contact area between the liquid and inner surface of the pipette tip (Figure 3 ). The separated surface charge density was calculated as $\sim 4.5 \times 10^{-6} \mathrm{C} / \mathrm{m}^{2}$ for deionized water. To confirm whether the charges can be totally transferred during dispensing, the surface charge density of the inner surface of the pipette tip of before dispensing must be known. However, the surface charge density is difficult to measure by conventional methods such as the streaming current $^{17}$ and electroosmosis methods ${ }^{18}$ due to the short length and tapered shape of the tips, so the surface charges of pipette tips before and after dispensing could not be measured. But the separated surface charge density is much smaller than the surface charge density of general materials like glasses, metal oxides, and fluoropolymers (order of $\left.10^{-2} \sim 10^{-3} \mathrm{C} / \mathrm{m}^{2}\right)^{19-21}$. Thus, we presume that the charge in the EDL is partially transferred during dispensing.

Although the charge may be partially transferred, the electrostatic interaction between the separated charges can affect pipetting. Small droplets are difficult to detach from the pipette tip during pipetting. Generally, this is thought to be due to the weak gravitational force compared with the capillary force between the droplet and the pipette tip. Here, we suggest that dispensing a small droplet is also

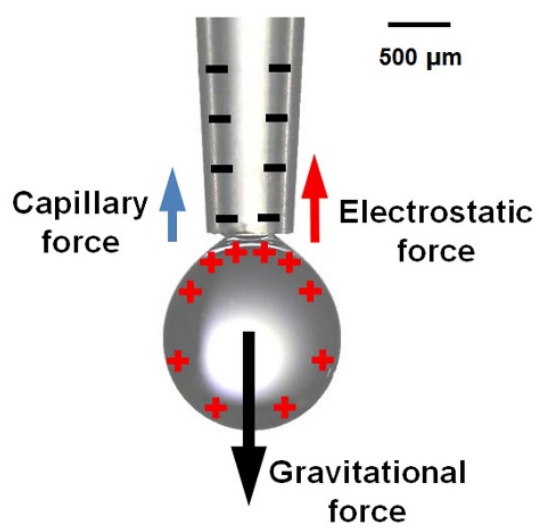

Figure 7 | Exerted forces on the charged droplet hanging on the pipette tip. When the charged droplet is hanging on the pipette tip, capillary, electrostatic and gravitational forces are exerted on the charged droplet. affected by the electrostatic interaction between the separated charges. The electrostatic force on the droplet is in direction opposite to that of the gravitational force (Figure 7), so as electrostatic force acting on the droplet increases, detaching the droplet becomes more difficult. Therefore we performed experiments to investigate how much the electrostatic interaction affects the detachment. By changing the $\mathrm{pH}$ of the solutions, the separated surface charge density was varied from $1.2 \times 10^{-6}$ to $5.6 \times 10^{-6} \mathrm{C} / \mathrm{m}^{2}$. The critical volume, at which the droplets start to detach spontaneously, increased as the separated surface charge density increased (Figure 8a); this result was confirmed by numerical analysis which considered the electrostatic interaction.

For dispensing small droplets or solutions of high viscosity using a pipette, reverse pipetting, in which the sequence of operations in the pipetting process is reversed, is recommended ${ }^{22}$. Reverse pipetting reduced the charge to one-fourth of that obtained using forward pipetting when dispensing droplets of the same volume (Supplementary Table S2). This is thought to be due to the charge accumulation at the upper interface by the electrostatic interaction between the separated charges. The accumulated charge would be in the liquid which remained in the tip. This reduce of the droplet charge by reverse pipetting could be another reason for allowing us to dispense a small droplet more easily than in forward pipetting.

\section{a}

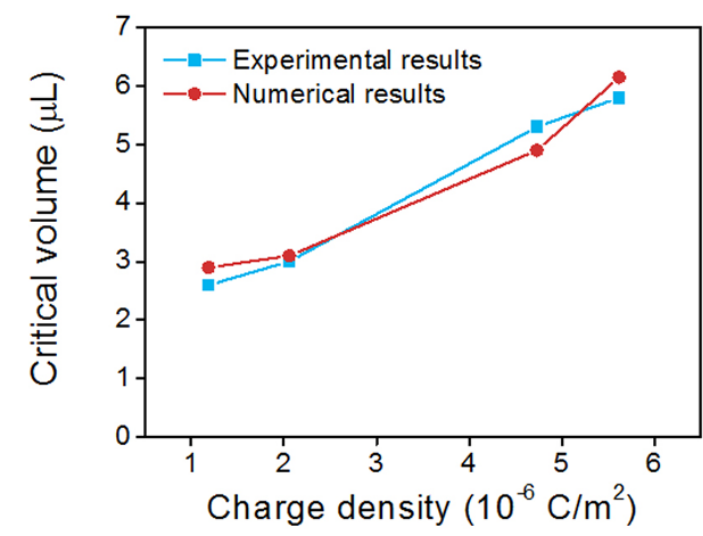

b

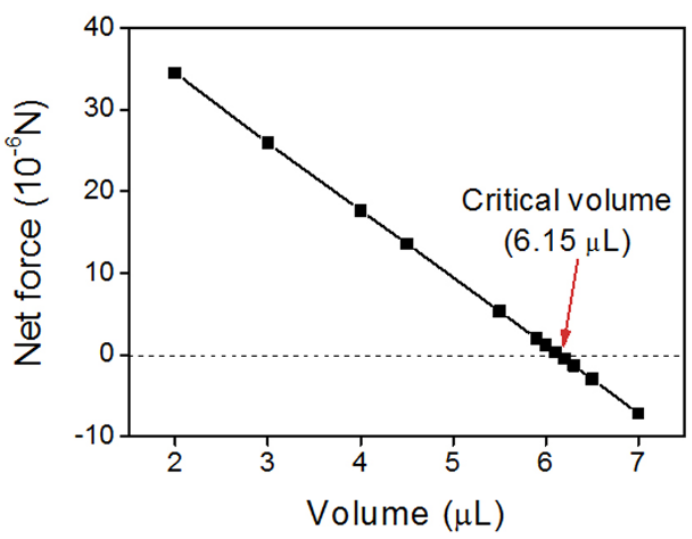

Figure $8 \mid$ Numerical simulation results to find the critical volume of a charged droplet. (a) Comparison of critical volumes obtained by numerical simulation and experiments. (b) Simulated result of the relationship between the volume of the charged droplet and the exerted net force (capillary + electrostatic - gravitational force) $(\mathrm{KCl} 0.01 \mathrm{M}, \mathrm{RH}=$ $30 \%)$. 
The effects of droplet charge can be important in experiments in which a pipette is used to dispense droplets. First, a charge on a droplet can decrease its surface tension. Because charges in electrolyte solutions repel each other, the charges on a droplet are distributed on the droplet surface. The repulsion between the charges on the surface of the droplet is opposed by its surface tension and effectively reduces the surface tension ${ }^{23}$. For a $7-\mu \mathrm{L}$ deionized water droplet $(\mathrm{RH}=$ $70 \%$ ), the amount of charge was $2.1 \times 10^{-10} \mathrm{C}$. This charge amount reduces the surface tension of the droplet from $72 \mathrm{mN} / \mathrm{m}$ to $67 \mathrm{mN} /$ $\mathrm{m}^{23}$; this difference of $\sim 7 \%$ might cause errors in experiments in which surface tension is important. Also, this charge effect on the surface tension can cause the instability of charged droplet ${ }^{24}$ and Coulombic fission when droplets evaporated ${ }^{25,26}$. Second, the charge can also affect the chemical stability of emulsions due to interactions with charged molecules such as ionic surfactants ${ }^{27,28}$ and can influence the combination and localization of charged bio-molecules such as proteins and DNAs ${ }^{29-31}$.

In summary, we have reported the previously unrecognized electrification phenomenon of a dispensed droplet in conventional pipetting. The magnitude of droplet charge showed clear dependency on $\mathrm{pH}$, concentration of electrolytes, and the coating material of pipette tip, and relatively weak dependency on atmospheric humidity. Contrary to common expectation, results suggest that this natural electrification of a droplet originates not from pre-developed charges on pipette tips but from the charge separation between a droplet and pipette tip surface. Because the present findings are unrecognized and undesired, the effects of this electrification phenomenon should be evaluated and discussed by researchers in various fields.

\section{Methods}

Charge measurement methods. The Faraday cup (FC) and droplet trajectory (DT) methods were used to measure the amount of charge. In the FC method, the charge was directly measured using a Faraday cup connected to an electrometer (Keithley Model 6517A). The Faraday cup was composed of two cylindrical electrodes separated by an insulator. When the charged droplet was dispensed into the inner electrode, to satisfy electric neutrality, the counter charge was transferred to the inner electrode from the electrometer. The total charge was measured by integrating the current from the electrometer ${ }^{32}$. In the DT method, charge was calculated from the trajectory of the droplet in dielectric oil. Freely falling droplets were dispensed using a pipette in silicone oil; they were deflected by an external uniform electric field applied between the two parallel planar electrodes placed $70 \mathrm{~mm}$ apart (Figure 1b). This field was generated using a function generator (Agilent Model 33220A) and a voltage amplifier (Trek Model 610E). The pathways of the droplets were recorded using a camera (Canon EOS 500D with MP-65E macro lens) through a window located midway between the two electrodes; Labview ${ }^{\mathrm{TM}}$ was used to calculate the velocities of the droplets by image processing. The location of the window was fixed for all experiments to minimize the non-uniformity of electric field due to the fringe effect and the effect of image charge near the metal electrodes. The applied voltages are adjusted according to the size and the charge of the droplets to locate the pathway of the droplets in the window (Supplementary Table S3). The applied voltage did not affect the charge measurement (Supplementary Table S4). Using the HadamardRybczynski solution for the drag force of a droplet, the charge of the droplet is estimated from force balance between the electrical and drag forces ${ }^{33}$. The two methods were performed selectively according to experimental cases. To reduce measurement error, the experiments were performed 8 or 20 times for each case. When the dependence of the parameters was measured (e.g., droplet volume, solution concentration and $\mathrm{pH}$, only one rack (96 tips) of the pipette tips was used for each set of the experiments to minimize any effect due to the difference between racks of tips. Therefore, the experiments were conducted only 8 times for each condition.

Preparation for accurate measurement. During the measurements, several factors should be considered to ensure accuracy. The movement of droplets can be affected by the static charge on surrounding objects like the acrylic chamber. Therefore, the number of objects located near the measurement system was minimized and the chamber was washed with isopropyl alcohol to remove the static charge of the chamber ${ }^{34}$. Electrokinetic effects of the oil and the aqueous solutions were minimized in the reservoir from which droplets were taken by pipetting. Because the oil also contains ions that can develop an EDL, the electrodes were frequently grounded to prevent it from reducing the electric field strength between the electrodes. The aqueous solution in the reservoir was also grounded because a charge of the opposite polarity can be accumulated by withdrawing charge from the reservoir during pipetting. The oil was replaced frequently to minimize any effects of contamination.

Materials. Highly viscous silicone oil (Shin-Etsu, KF96 $50 \mathrm{cSt}$ ) was used as the suspending medium to ensure that the falling velocity of the droplets was in the low
Reynolds number regime. Deionized water or electrolyte solutions such as phosphate buffered saline, and $\mathrm{NaCl}$ or $\mathrm{KCl}$ solutions were used as the droplet phase. The droplet volumes ranged from 2 to $10 \mu \mathrm{L}$, corresponding to diameters of 1.6 to $2.7 \mathrm{~mm}$. When the effect of the $\mathrm{pH}$ of the solutions was investigated, $\mathrm{HCl}$ and $\mathrm{KOH}$ solutions were used to regulate $\mathrm{pH}$. Micropipette tips $(1-10 \mu \mathrm{L})$ from three manufacturers were used to dispense droplets.

Electroosmosis in pipette tips. To apply an electric field inside a pipette tip filled with electrolyte, $\mathrm{Ag} \mid \mathrm{AgCl}$ electrodes, free from bubble formation, were used. One of the electrodes was pinned to the narrow end of the pipette tip and the other was placed at the center of the wide end of the pipette tip (Supplementary Figure S1a). To visualize the flow inside the pipette tip, a little Rhodamine $6 \mathrm{G}$ solution was injected through the open end of the tip. To minimize thermal effects on the flow, $\mathrm{KCl}$ solution of low concentration was chosen because of its low electrical conductivity, and the applied voltage was $<30 \mathrm{~V}$

Numerical simulation (Supplementary Figure S1b) of the electroosmotic flow in micropipette tips was performed using commercial finite element software, COMSOL Multiphysics ${ }^{\circledR}$. The Smoluchowski slip velocity condition ${ }^{35}$ (Equation 1) was given to the inner surface of the pipette tip:

$$
U_{\text {Slip }}=-\frac{\varepsilon \zeta E_{t}}{\mu}
$$

where $\zeta$ is the zeta potential of the pipette tip surface, $E_{t}$ is the tangential electric field strength, $\varepsilon$ is the electrical permittivity of the solution and $\mu$ is its viscosity. Values of $\mathrm{E}_{t}$ and $\zeta$ were chosen arbitrarily. Simulation results were compared qualitatively with experimental results.

Charge effect on the detachment of droplets from pipette tips. To investigate the charge effect on pipetting itself, experiments were performed to find the critical volume at which a droplet drops naturally from a pipette tip. When the size of the droplet hanging on the pipette tip exceeds this volume, the droplet is detached by gravitational force which overcomes the combination of capillary force and electrostatic force. The electrostatic force is exerted between the charged droplet and an unlike-charged pipette tip. To vary the charge of the droplet, the $\mathrm{pH}$ dependence of the charge was exploited. The charges of the droplets from the solutions of different $\mathrm{pH}$ were measured before the experiments. The procedure of the experiment was as follows. The solution was aspirated from the reservoir to the pipette tip using a syringe pump (Harvard Apparatus, Pump 11 Elite). Then a great part of the solution was infused with fast dispensing speed $(\sim 10 \mu \mathrm{L} / \mathrm{min})$ to avoid evaporation. The last moment of the infusing was controlled very slowly to minimize perturbation caused by injection pressure. The experiments were conducted 10 times for each droplet volume. The critical volume was determined as the minimum volume at which the droplet fell naturally more than 5 times, (i.e., in half of the performed experiments). The net force on the charged droplet (capillary + electrostatic - gravitational force) was calculated. To calculate the capillary force, the surface tension of the droplet was assumed to be $72 \mathrm{mN} / \mathrm{m}$. The electrostatic force, $F_{\mathrm{E}}$, was numerically calculated using $\mathrm{z}$-coordinate integration of the electrostatic force on the droplet.

$$
F_{E}=\int_{S} \sigma_{s} E_{z} d S,
$$

Where $\sigma_{s}$ and $E_{z}$ are the surface charge density of the droplet and z-component of the electric field, respectively.

Numerical simulation to evaluate the electrostatic force on the charged droplet was performed using commercial finite element software, COMSOL Multiphysics ${ }^{\circledR}$. The surface charge density $\sigma_{0}$ of the inner surface of the pipette tip was assumed to be constant. The values of the surface charge density were calculated from the experimental results. The electrical potential $V_{0}$ of the droplet was adjusted to give the measured charge amount on the droplet. Ground conditions were applied to the outer boundaries, which are located far from the droplet. The numerical domain size, D, was set by considering the effect of the outer boundaries and the increasing numerical cost due to enlargement of the domain size (Supplementary Figure S2a, b).

1. Berthier, E., Warrick, J., Casavant, B. \& Beebe, D. J. Pipette-friendly laminar flow patterning for cell-based assays. Lab. Chip. 11, 2060-2065 (2011).

2. McPherson, A. L. \& Walker, G. M. A microfluidic passive pumping Coulter counter. Microfluid. Nanofluid. 9, 897-904 (2010).

3. Ainla, A., Jansson, E. T., Stepanyants, N., Orwar, O. \& Jesorka, A. A Microfluidic Pipette for Single-Cell Pharmacology. Anal. Chem. 82, 4529-4536 (2010).

4. Jessen, M. Tips for Reducing Static Electricity. (Bioscience Technology, 2011).

5. Im, D. J., Noh, J., Moon, D. \& Kang, I. S. Electrophoresis of a charged droplet in a dielectric liquid for droplet actuation. Anal. Chem. 83, 5168-5174 (2011).

6. Im, D. J., Noh, J., Yi, N. W., Park, J. \& Kang, I. S. Influences of electric field on living cells in a charged water-in-oil droplet under electrophoretic actuation. Biomicrofluidics 5, 044112 (2011).

7. Khayari, A. \& Pérez, A. T. Charge acquired by a spherical ball bouncing on an electrode: Comparison between theory and experiment. IEEE Trans. Dielectr. Electr. Insul. 9, 589-595 (2002).

8. Masliyah, J. H. \& Bhattacharjee, S. Electrokinetic and Colloid Transport Phenomena. (John Wiley \& Sons, Inc., 2005).

9. Hunter, R. J. Foundations of Colloid Science. (Oxford University Press, 2001). 
10. Lyklema, J. Fundamentals of Interface and Colloid Science. (Academic Press, 1995).

11. Kirby, B. J. \& Hasselbrink, E. F. Jr. Zeta potential of microfluidic substrates: 1 Theory, experimental techniques, and effects on separations. Electrophoresis 25, 187-202 (2004).

12. Israelachvili, J. N. Intermolecular and Surface Forces. (Academic Press, 1992).

13. de Gennes, P. G. Wetting: Statics and dynamics. Rev. Mod. Phys. 57, 827-863 (1985).

14. Digilov, R. Charge-induced modification of contact angle: The secondary electrocapillary effect. Langmuir 16, 6719-6723 (2000).

15. Buehrle, J., Herminghaus, S. \& Mugele, F. Interface profiles near three-phase contact lines in electric fields. Phys. Rev. Lett. 91, 086101 (2003).

16. Gaskell, S. J. Electrospray: Principles and practice. J. Mass. Spectrom. 32, 677-688 (1997).

17. Werner, C., Zimmermann, R. \& Kratzmüller, T. Streaming potential and streaming current measurements at planar solid/liquid interfaces for simultaneous determination of zeta potential and surface conductivity. Colloid and Surfaces A: Physicochem. Eng. Aspects 192, 205-213 (2001).

18. Arulanandam, S. \& Li, D. Determining $\zeta$ potential and surface conductance by monitoring the current in electro-osmotic flow. J. Colloid Interface Sci. 225, 421428 (2000).

19. Kosmulski, M. Chemical Properties of Material Surfaces. (Dekker, Newyork, 2001).

20. Preočanin, T. et al. Surface charge at Teflon/aqueous solution of potassium chloride interfaces. Colloids and Surfaces A: Physocochem. Eng. Aspects 412, 120 128 (2012).

21. Zimmermann, R., Dukhin, S. \& Werner, C. Electrokinetic measurements reveal interracial charge at polymer films caused by simple electrolyte ions. J. Phys. Chem. B 105, 8544-8549 (2001).

22. Ewald, K. Impact of Pipetting Techniques on Precision and Accuracy. (Eppendorf AG).

23. Davis, E. J. \& Bridges, M. A. The Rayleigh limit of charge revisited: Light scattering from exploding droplets. J. Aerosol Sci. 25, 1179-1199 (1994).

24. Rayleigh, L. On the equilibrium of liquid conducting masses charged with electricity. Phil. Mag. 14, 184-186 (1882).

25. Smith, J. N., Flagan, R. C. \& Beauchamp, J. L. Droplet evaporation and discharge dynamics in electrospray ionization. J. Phys. Chem. A 106, 9957-9967 (2002).

26. Duft, D. et al. Rayleigh jets from levitated microdroplets. Nature 421, 128 (2003).

27. Choi, S. J., Decker, E. A., Henson, L., Popplewell, L. M. \& McClements, D. J. Influence of droplet charge on the chemical stability of citral in oil-in-water emulsions. J Food Sci 75, C536-C540 (2010).

28. Gu, Y. G. \& Li, D. Q. Electric charge on small silicone oil droplets dispersed in ionic surfactant solutions. Colloid Surface A 139, 213-225 (1998).
29. Yeung, T. et al. Membrane phosphatidylserine regulates surface charge and protein localization. Science 319, 210-213 (2008).

30. Segura, T. \& Shea, L. D. Materials for non-viral gene delivery. Ann Rev Mater Res 31, 25-46 (2001)

31. Xu, X. N. \& Yeung, E. S. Long-range electrostatic trapping of single-protein molecules at a liquid-solid interface. Science 281, 1650-1653 (1998).

32. Low-Level Measurements Handbook. (Keithley Instruments, 2006).

33. Leal, L. G. Advanced Transport Phenomena: Fluid Mechanics and Convective Transport Processes. (Cambridge University Press, 2007).

34. Ohmi, T., Sudoh, S. \& Mishima, H. Static charge removal with IPA solution. Semiconductor Manufacturing, IEEE Transactions on 7, 440-446 (1994).

35. Probstein, R. F. Physicochemical Hydrodynamics. (John Wiley \& Sons, 1994).

\section{Acknowledgements}

This research was supported by Basic Science Research Program grant No. 2013010483 through the National Research Foundation of Korea (NRF) funded by the Ministry of Education, Science and Technology (MEST). This work was also supported by the NRF grant No. 2012R1A2A2A06047424 and Mid-career Researcher Program No. 2011-0029454 funded by the Korea government (MEST).

\section{Author contributions}

D.J.I. and K.H.K. conceived and supervised the research. D.J.I. and H.L. designed the experiments. D.C. performed the experiments. D.J.I., H.L. and D.C. analyzed the experimental data. D.J.I., H.L. and I.S.K. wrote the manuscript. G.L. and D.S.K. advised and supervised the experiments. All authors discussed the results and commented on the manuscript.

\section{Additional information}

Supplementary information accompanies this paper at http://www.nature.com/ scientificreports

Competing financial interests: The authors declare no competing financial interests. How to cite this article: Choi, D. et al. Spontaneous electrical charging of droplets by conventional pipetting. Sci. Rep. 3, 2037; DOI:10.1038/srep02037 (2013).

(c) $($ ) This work is licensed under a Creative Commons AttributionBY NC No NonCommercial-NoDerivs 3.0 Unported license. To view a copy of this license, visit http://creativecommons.org/licenses/by-nc-nd/3.0 